\title{
COLESTEROL E COMPOSIÇÃO DOS ÁCIDOS GRAXOS NAS DIETAS PARA HUMANOS E NA CARCAÇA SUÍNA
}

\author{
FATTY ACIDS CONCENTRATION AND LEVEL OF CHOLESTEROL \\ IN DIETS FOR HUMANS AND PRESENT IN SWINE CARCASSES
}

\author{
Maria do Carmo Mohaupt Marques Ludke ${ }^{1}$ Jorge López $^{2}$
}

- REVISÃO BIBLIOGRÁFICA -

RESUMO

O colesterol é uma substância complexa do tipo lipídio-esteróide presente principalmente nas gorduras animais. Apresenta múltiplas funções no organismo, entretanto, problemas no metabolismo do colesterol no organismo podem acarretar aumento na sua concentração no sangue e conseqüentemente doenças coronárias como arterosclerose. Porém, já está comprovado que o consumo de colesterol é um fator de risco para pessoas que apresentam problemas genéticos de regulação do seu metabolismo. São pessoas que possuem níveis de colesterol acima de 200mg/dl e com concentração de HDL no sangue inferior a $35 \mathrm{mg} / \mathrm{dl}$. Apenas para estes indivíduos é importante o controle do colesterol através da dieta, na qual o consumo de carne suína, como de qualquer carne de outra espécie animal, deve ser considerada a composição dos ácidos graxos da gordura subcutânea e intramuscular. O fornecimento de dietas para suínos com maior concentração de ácidos graxos poliinsaturados apresentaram maior teor deste tipo de gordura nas suas carcacas. A suplementação destas gorduras de origem vegetal não pode ultrapassar a $4 \%$ nas dietas, devido à obtenção de carcaças com deficiência de conservação, apesar de originar uma carne mais benéfica à saúde do consumidor.

Palavras-chave: alimento, arterosclerose, carne suína, colesterol, gorduras saturada e poliinsaturada.

\section{SUMMARY}

Cholesterol is a complex lipid-steroid like substance mostly present in animal fat, and it has many essencial functions in living organisms. However, in humans, any metabolic problems can increase blood cholesterol concentration and, consequently, increase the risk of coronary heart disease, such as arteriosclerosis. It is a known fact that cholesterol consumption is a risk factor for people who have genetic disorders in the metabolic regulation of cholesterol. These people have plasma levels of cholesterol above $200 \mathrm{mg} / \mathrm{dl}$ and a plasma concentration

\begin{abstract}
of high density lipoprotein (HDL) under $35 \mathrm{mg} / \mathrm{dl}$. For these people, it is important to control the cholesterol level in the diet. The consumption of pork meat as well as any kind of meat, must be monitored for the fatty acid composition the intramuscular and subcutaneous fat. Swine that received diets with high concentration of polyunsaturated fats present higher concentrations of these fatty acids in the carcass. The supplementation of swine diets with polyunsaturated fatty acids of vegetable origin cannot be above 4\%, due to the detrimental effect on the conservation of the carcasses, even though it results in healthier meat for human consumption.
\end{abstract}

Key words: arteriosclerosis, cholesterol, feed, pork meat, saturated and polyunsaturated fatty acids.

\section{INTRODUÇÃO}

O colesterol é uma substância complexa que apresenta inúmeras funções no organismo, porém, ocorrendo problemas no seu metabolismo pode acarretar aumento em sua concentração no sangue e, conseqüentemente, doenças coronarianas como arterosclerose, além de causar hipertensão arterial, problemas de diabete mellitus e formação de cálculos biliares. Segundo STEHBENS (1989), os fatores de risco mais importantes são: sexo masculino, idade avançada, hipertensão, fumo, diabetes, baixa atividade física, alto consumo de gordura e colesterol e, principalmente, o histórico familiar (genética). Este último fator é considerado o principal, devido aos fatores de risco nas pessoas com tendência à hipercolesterolemia serem mais proeminentes, obrigando estes indivíduos a serem cautelosos na sua alimenta-

\footnotetext{
${ }^{1}$ Zootecnista, MSc. Aluna de Doutorado, Departamento de Zootecnia da Universidade Federal do Rio Grande do Sul (UFRGS), reside atualmente na rua Independência, 283/apt. 304, bloco A, Concórdia - SC, 89700-000. Autor para correspondência.

${ }^{2}$ Agrônomo, PhD. Professor Titular do Departamento de Zootecnia da UFRGS. Porto Alegre - RS. E-mail: dini@ez-poa.com.br Recebido para publicação em 17.04.98. Aprovado em 12.08.98
} 
ção. Por isso, o uso de alimentos com altos níveis de colesterol tem sido condenado pela maioria dos médicos. Este fato tem provocado reação no consumidor, que passa a evitar o consumo de alimentos nutritivos como por exemplo, a carne suína que não apresenta teor de colesterol maior do que a carne bovina ou a carne de frango. $\mathrm{O}$ problema maior não está relacionado diretamente com a carne, mas ao tipo de gordura (monoinsaturada, poliinsaturada ou saturada) que está presente na dieta. Segundo KINSELLA et al. (1990), a relação entre o consumo de gorduras saturadas e insaturadas com os níveis de colesterol no soro sangüíneo do homem sugere implicações evidentes.

Cerca de $70 \%$ da gordura do suíno localiza-se abaixo da pele e não entre as fibras musculares, que é considerada a pior (SILVA, 1995). Assim, nem todas as gorduras animais são metabolicamente equivalentes e alguns lipidios, de origem animal são potencialmente benéficos para a saúde (COBOS $\boldsymbol{e t}$ al., 1994 ). Baseado nestes fatos, elaborou-se a presente revisão bibliográfica sobre o colesterol e seu significado biológico para a vida animal, na tentativa de minimizar as dúvidas sobre este fator de risco e orientar, de forma imparcial, as pessoas na preservação da saúde humana, sempre sob o enfoque de respeito aos setores agropecuários envolvidos na atividade de produção de proteína animal para um consumo nutricionalmente equilibrado.

\section{IMPORTÂNCIA DO COLESTEROL}

O colesterol é uma substância importante para manter a saúde e diversas funções no organismo animal. Sem ele todos os animais, inclusive o homem, poderiam morrer, razão pela qual ele é exigido por todos os tecidos e células vivas do corpo. Assim, suas principais funções são: componente da célula que desempenha uma importante função estrutural e funcional na membrana plasmática, assim como nas membranas das organelas internas da célula; síntese de ácidos biliares que participam da emulsificação, digestão e absorção de lipídios e vitaminas lipossolúveis, no intestino delgado; síntese de hormônios esteróides; síntese da vitamina E e componente da pele que, junto a outros materiais gordurosos, torna-a altamente resistente à absorção de água e outras substâncias hidrossolúveis, que, se absorvidas, podem causar danos ao organismo. Além disso, o colesterol e outros componentes da pele têm função de prevenir contra perdas excessivas de água por evaporação, o que acarretaria problemas de desidratação e morte.

\section{METABOLISMO DO COLESTEROL}

A maior parte do colesterol do organismo, segundo MILES (1989) aproximadamente 75\%, origina-se da biossíntese (colesterol endógeno), enquanto apenas $25 \%$ é fornecido pela dieta (colesterol exógeno). Quando a alimentação é rica em colesterol, ocorre um bloqueio da sua síntese endógena. Por outro lado, a redução muito acentuada de colesterol-alimento pode aumentar sua síntese endógena (HARPER, 1993). O colesterol na luz intestinal pode ter sua origem, além da dieta, também da bile que carreia 1 a $2 \mathrm{~g} /$ dia, e das secreções ou da descamação das células da mucosa intestinal. Na ausência da bile, não ocorrerá absorção do colesterol.

Mecanismos regulatórios devem existir para balancear a taxa de síntese de colesterol dentro do corpo contra sua taxa de excreção (CHAMPE \& HARVEY, 1994). Segundo os autores, um desbalanço nesta regulação pode conduzir a uma elevação na circulação dos níveis de colesterol no plasma, causando então a doença da artéria coronária, enquanto secreção excessiva na bile resulta em precipitação do colesterol no ducto biliar, causando a doença denominada colelitíase. Através da HMG-CoA redutase, a enzima marcapasso na síntese do colesterol, há diferentes tipos de controles metabólicos, dentre eles destacam-se :

- inibição por feedback $\rightarrow \mathrm{O}$ colesterol é um inibidor por feedback da HMG-CoA redutase, ocasionando diminuição da síntese do colesterol.

- regulação hormonal $\rightarrow$ Através do complexo cascata de atividade e inibição da enzima semelhante a regulação de síntese de glicogênio, nos quais sofrem efeito dos hormônios insulina e glucagon.

- inibição por drogas $\rightarrow$ Lovastatina e Mevastatina são inibidores competitivos da HMGCoA redutase. Elas são utilizadas para pacientes com hipercolesterolemia.

O colesterol é transportado no plasma pelas lipoproteínas que são sintetizadas no fígado e intestino. Segundo KRIS-ETHERTON et el. (1988), as lipoproteínas do plasma sangüineo são classificadas de acordo com suas densidades em 5 grandes grupos: Quilomicron (QM), Lipoproteínas de densidade muito baixa (Very Low-Density Lipoproteins, VLDL), Lipoproteínas de densidade intermediária (Intermediate-Density Lipoproteins, IDL), Lipoproteínas de densidade baixa (Low-density lipoproteins, LDL) e Lipoproteinas de densidade alta (Hightdensity lipoproteins, HDL). O LDL constitui o principal componente do pool de ésteres de colesterol no corpo humano, por isto este componente é freqüen-

Ciência Rural, v. 29, n. 1, 1999. 
temente referido como "mau colesterol" (SMITH \& PINCKNEY, 1991). Porém, o HDL são as mais densas partículas das lipoproteínas plasmáticas, é o "bom colesterol", pois apesar de ser constituído de $15 \%$ de ésteres de colesterol, ele apresenta apenas $4 \%$ de triglicerídeos. Além disso, ele atua como a principal via de degradação do colesterol pela conversão a ácidos biliares no fígado. Estes são liberados no intestino delgado, onde auxiliam na absorção dos lipídios para formarem os quilomicrons. Os ácidos biliares são reabsorvidos durante a absorção lipídica. Então, as principais vias de excreção do colesterol do corpo são: (1) conversão a ácidos biliares, no qual é excretado nas fezes, onde é formado o coprostanol (principal esterol das fezes) por ação microbiana; (2) secreção de colesterol na bile, no qual é transportado ao intestino para eliminação e, (3) uma fração menor do colesterol é convertida em hormônios esteróides, sofrendo eliminação urinária.

\section{COLESTEROL, CARDIOPATIA CORONÁRIA E ARTEROSCLEROSE}

Muitos pesquisadores demonstraram uma correlação entre os níveis elevados de lipídios séricos, principalmente o colesterol, e a incidência de cardiopatia coronária e arterosclerose (KEYS, 1970; ANDERSON et al., 1973). Para ANDERSON $\boldsymbol{e t}$ al. (1976), os níveis de colesterol no soro não dependiam somente do conteúdo de colesterol dos alimentos, mas também do balanço entre ácidos graxos saturados (AGS) e ácidos graxos poliinsaturados (AGP). Segundo HARPER (1993), deve haver restrições quanto ao consumo de colesterol apenas em indivíduos que possuem problemas no metabolismo de colesterol no organismo. Só ocorrem mudanças no nível de colesterol no plasma em indivíduos normais, caso haja um consumo de 400 - 500mg de colesterol / dia (HOPKINS, 1992). Com isso, conclui-se que indivíduos que, continuamente, têm níveis elevados de colesterol no sangue são os que apresentam hipercolesterolemia familiar, por possuir uma disfunção genética herdada pelos seus antecedentes, através de uma cópia de um gene mutante que não permite o organismo regular corretamente o colesterol.

Pessoas com colesterol total de 200 $239 \mathrm{mg} / \mathrm{dl}$, mesmo sem doença cardíaca coronariana (DCC) definida, mas com outros fatores de risco (sexo masculino, histórico familiar com DCC, fumo, hipertensão, diabete mellitus e obesidade) necessitam informações sobre dieta alimentar e dosagem anual do colesterol total. Segundo REISER (1989), cerca de $75 \%$ da população de homens de meia- idade não respondem à dieta de colesterol, ou seja, não apresentam hipercolesterolemia familiar. Eles têm mecanismos homeostáticos para resistir ou adaptar-se aos fatores dietéticos. Conseqüentemente, apenas $25 \%$ da população necessita preocupar-se com o nível de colesterol e deve estabelecer uma meta de alimentação hipocolesterolêmica.

\section{CONTROLE DO COLESTEROL ATRAVÉS DA DIETA}

Indivíduos com colesterol entre 200 $239 \mathrm{mg} / \mathrm{dl}$ e que apresentem dois ou mais fatores de riscos a DCC devem iniciar um controle com uma dieta que restringe a ingestão de colesterol e o total de gorduras, particularmente gorduras saturadas. Segundo o NCEP (1988) uma dieta recomendada para este caso deve possuir a composição mostrada na Tabela 1.

Estudos compararam níveis de colesterol no sangue de indivíduos vegetarianos e nãovegetarianos, no qual SMITH \& PINCKNEY (1991) encontraram altos níveis de colesterol em ambos os indivíduos. Porém, os não-vegetarianos apresentaram 292mg de colesterol no sangue contra 206mg dos vegetarianos. Os primeiros consumiram $36 \%$ a mais de gordura, e este foi o motivo da discrepância entre os dois, afirmaram os autores.

Segundo NORUM (1992), a quantidade e o tipo de gordura na dieta têm efeito nas concentrações de lipídios no sangue. $\mathrm{O}$ autor ressalta que a conclusão de vários trabalhos realizados é que os ácidos graxos saturados (AGS) aumentam e os ácidos graxos poliinsaturados (AGP) diminuem a concentração de colesterol no plasma, sendo que o ácido graxo monoinsaturado (ácido oléico) e o ácido esteárico saturado são neutros. Porém, a resposta ao consumo de ácidos graxos saturados (AGS) sobre a

Tabela 1 - Dieta recomendada pelo "National Cholesterol Education Program".

\begin{tabular}{lc}
\hline \multicolumn{1}{c}{ Constituintes } & $\%$ Caloria Total \\
\hline Gordura total & $<30 \%$ \\
Saturada & $<10 \%$ \\
Monoinsaturada & $10-15 \%$ \\
Poliinsaturada & $>10 \%$ \\
\hline Colesterol & $<300 \mathrm{mg} / \mathrm{d}$ \\
\hline
\end{tabular}




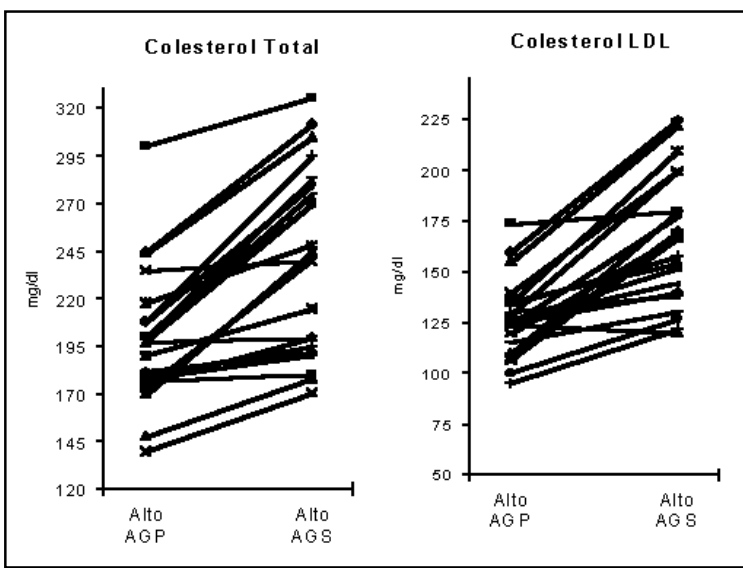

Figura 1 - Resposta na concentração de colesterol total e LDL em indivíduos consumindo dois tipos de gorduras. Fonte: GRUNDY \& VEGA (1988)

concentração de colesterol no plasma varia de indivíduo para indivíduo, como mostrado na Figura 1. Alguns indivíduos aumentam a concentração de colesterol e LDL no sangue quando o tipo de gordura no alimento é modificado, enquanto em outros indivíduos a resposta fica inalterada (GRUNDY \& VEGA, 1988).

O tipo e quantidade de gordura na dieta também afetará a concentração de triglicerídeos. Os ácidos graxos ômega-3, presentes em óleos de peixes, parecem serem mais eficientes em baixar a concentração de triacilgliceróis no plasma do que os óleos vegetais, geralmente ricos em ácidos graxos ômega-6 (CONIGLIO, 1992). Dentre os mecanismos sugeridos como possíveis responsáveis para o efeito dos óleos de peixes nos níveis de triacilglicerol no plasma são inibição da síntese e secreção de triacilglicerol, contendo lipoproteínas, inibição de síntese de ácido graxo, aumento da oxidação do ácido graxo, diminuição da atividade de enzimas responsáveis para esterificação de ácido graxo e mudança na relação dos ésteres de ácidos graxos formados (CONIGLIO, 1992). Além disso, as gorduras poliinsaturadas são também mais rapidamente diluídas do que a gordura saturada, provavelmente, devido ao aumento da susceptibilidade dos quilomicron poliinsaturado a lipase lipoprotéica (WEINTRAUB et $\boldsymbol{a l} .$, 1988). De acordo com STURDEVANT $\boldsymbol{e}$ t al. (1973), a gordura poliinsaturada pode aumentar também a secreção de ácidos biliares e colesterol na bile pela elevação do número de receptores LDL, permitindo a não circulação desta lipoproteína no plasma. Além disso, segundo BEYNEN \& KATAN (1985), este tipo de gordura quando ingerida vai sintetizar no fígado mais corpos cetônicos do que triglicerídeos, diminuindo assim a quantidade de lipoproteínas LDL-colesterol no sangue. Esta última justificativa sobre o efeito da gordura poliinsaturada em reduzir a concentração do "mau colesterol" na circulação sangüínea, foi confirmada por BUDOWSKI (1981). O autor afirmou que a presença de ligações duplas apresentam maior possibilidade de uma $\gamma$-oxidação, que vai formar o composto Propionil-CoA, no qual tem efeito cetogênico.

A maioria das gorduras de origem animal (bovinos, suínos e aves) como as das carnes, leite e ovos são ricas em ácidos graxos saturados e monoinsaturados, porém pobres em ácidos graxos poliinsaturados, assim recomenda-se que as gorduras saturadas dos animais devam ser substituídas, em parte, pelas gorduras poliinsaturadas, quando há necessidade de controlar ou reduzir o nível de colesterol no sangue, como pode ocorrer com indivíduos hipercolesterolêmicos. Porém, é importante também estes mesmos indivíduos consumirem fontes de proteínas, como a carne, com isso um grande número de pesquisas mostraram que dietas compostas com gorduras insaturadas para animais não-ruminantes diminuem a concentração de gordura saturada e aumentam os níveis de gorduras insaturadas, propiciando melhor alternativa para a saúde do consumidor.

\section{INFLUENCIA DA DIETA SOBRE A COMPOSIÇÃO DOS ÁCIDOS GRAXOS DOS LIPÍDIOS DA CARCAÇA SUÍNA}

Os lipídios são imprescindíveis para a aceitabilidade da carne, já que sua concentração na mesma e a composição de cada uma das frações lipídicas influem consideravelmente nas propriedades organolépticas (textura, sabor, aroma, cor, etc). Além disso, atualmente, sabe-se que nem todas as gorduras animais são prejudiciais à saúde. Com isso, como se pode conseguir uma carne cuja gordura seja nutritivamente adequada?

O tipo de gordura da dieta constitui a maior fonte de variação na composição em ácidos graxos dos lipídios de depósito, de forma particular nos animais não-ruminantes. Muitos experimentos com suínos (BROOKS, 1971; VILLEGAS et al., 1973; SKELLEY et al., 1975; MORGAN et al., 1992) e com aves (ASGHAR $\boldsymbol{e t} \boldsymbol{a l}$. , 1990) têm mostrado que a adição de diferentes gorduras como sebo, óleo de soja, óleo de amendoim, óleo de linhaça, óleo de coco e manteiga de cacau numa dieta influem na composição em ácidos graxos dos lipídios da carne dos não-ruminantes. Dietas com quantidades adicionais de gordura, com elevado conteúdo em ácido linoléico, como óleo de soja ou óleo de amendoim, aumentam a quantidade de ácido aracdônico 
na gordura da carcaça dos suínos (MORGAN et $\boldsymbol{a l}$., 1992). Porém, em uma concentração maior de ácido linolênico na dieta ocasiona aumento dos ácidos graxos poliinsaturados da família W-3. Segundo IRIE \& SAKIMOTO (1992), ao adicionarem óleo de peixe na alimentação de suínos, aumentaram a quantidade de ácidos graxos W-3 na gordura dos animais, porém ocorreu diminuição na firmeza desta gordura. $\mathrm{O}$ uso de produtos de peixe para aumentar conteúdo de ácidos graxos poliinsaturados W-3 na carne e subprodutos parecem produzir problemas associados ao aroma e uma maior susceptibilidade dos produtos cárnicos à oxidação (PARK $\boldsymbol{e t} \boldsymbol{a l}$., 1989). Entretanto, MORGAN et al. (1992) adicionaram ácidos graxos W-3 nas dietas para suínos, provocando aumentos significativos destes ácidos graxos nas gorduras da carcaça dos animais, sem que houvesse efeitos adversos durante o tratamento da carcaça e sem modificação aguda nas características organolépticas durante o cozinhamento. Como vimos, o ácido linoléico e linolênico constituem 95 $97 \%$ dos ácidos graxos poliinsaturados, nos quais a sua quantidade na dieta é diretamente proporcional à gordura corporal (SHERF \& BIEBERWLASCHNY, 1990). Os autores também ressaltaram que, por ser ácido graxo poliinsaturado ocasiona pouca firmeza na espessura de toucinho, apenas quando sua concentração está acima de $150 \mathrm{mg} / \mathrm{g}$ de gordura. E só são encontrados estes valores quando esta gordura (óleos vegetais) na dieta exceder a $4 \%$.

A adição de óleo de canola, que possue um conteúdo relativamente alto em ácido oléico, nas dietas provoca efeitos adversos na qualidade da carne do suíno, com um amaciamento excessivo (MYER et al., 1992); além de maior susceptibilidade a oxidação lipídica. RHEE et al. (1988) utilizaram um óleo de uma variedade de girassol com maior conteúdo em ácido oléico e menor proporção de ácidos graxos poliinsaturados do que o óleo de canola. As carcaças dos suínos alimentados com dietas que continham $12 \%$ deste óleo de girassol foram ainda brandas, porém em menor proporção do que o óleo de canola. A adição de óleo de girassol de alta quantidade de ácido oléico na dieta produz um aumento na concentração deste ácido graxo e diminuição na proporção de ácido palmítico nos lipídeos da carcaça suína. Assim, as carcaças dos animais alimentados desta forma apresentam propriedades sensoriais aceitáveis (MILLER et al., 1990; RHEE et al., 1990; ZIPRIN et al., 1990).
Além das dietas, contendo ácidos graxos poliinsaturados para suínos, ocasionarem modificação na composição da sua gordura corporal, também reduz o teor de colesterol no organismo, como no ser humano, devido ao aumento da excreção do colesterol, via sais biliares (NABER, 1976). Foi confirmado por IBRAHIM \& McNAMARA (1988) ao adicionarem óleo de milho e banha nas dietas para suínos. Os autores encontraram um efeito significativo nos níveis de colesterol no plasma, ou seja, aqueles que consumiram óleo de milho apresentaram 34\% de redução na concentração de colesterol em relação aos que receberam banha. Os níveis de colesterol encontrados foram: $54 \pm 10 \mathrm{mg} / \mathrm{dl}$ vs $81 \pm 7 \mathrm{mg} / \mathrm{dl}$ nos animais alimentados com óleo de milho e banha, respectivamente. Em um trabalho realizado por LEAT et al. (1964), no qual foi adicionado $10 \%$ de óleo de milho em dietas semi-sintéticas para suínos, além de $10 \%$ de sebo e uma dieta controle, obteve mais alto teor de ácidos graxos poliinsaturados (linoléico) na gordura da carcaça dos animais do $1^{\circ}$ grupo. Os dados de gordura intermuscular e subcutânea dos animais pode ser visto na Tabela 2 .

\section{TEORES DE COLESTEROL NA CARCAÇA SUÍNA}

REITMEIER \& PRUSA (1987) observaram que o teor de colesterol para carne de porco crua foi maior com $18 \%$ e $23 \%$ de gordura. Os valores obtidos para carne com 4, 9, 18 e $23 \%$ de gordura foram respectivamente, 55,93; 57,49; 69,59 e $65,86 \mathrm{mg} / 100 \mathrm{~g}$. Valores semelhantes foram obtidos por BOHAC \& RHEE (1988) para diferentes músculos suínos : 55,9mg/100g para lombo ("Longissimus dorsi"); 53,1mg / $100 \mathrm{~g}$ para pernil ("semimem-
Tabela 2 - Composição dos ácidos graxos (\%) da gordura subcutânea e intermuscular da carcaça suína alimentados com as dietas: sem gordura, com sebo bovino ou com óleo de milho.

\begin{tabular}{|c|c|c|c|c|c|c|}
\hline \multirow{2}{*}{$\begin{array}{c}\text { Tipos de ácidos } \\
\text { graxos }\end{array}$} & \multicolumn{3}{|c|}{ SUBCUTÂNEA } & \multicolumn{3}{|c|}{ INTERMUSCULAR } \\
\hline & S/gordura & Sebo & $\begin{array}{l}\text { Óleo de } \\
\text { milho }\end{array}$ & S/gordura & Sebo & $\begin{array}{l}\text { Óleo de } \\
\text { milho }\end{array}$ \\
\hline Palmítico & 25,0 & 23,0 & 18,7 & 26,6 & 22,6 & 18,1 \\
\hline Esteárico & 14,5 & 10,5 & 11,5 & 13,8 & 10,3 & 10,5 \\
\hline Oléico & 53,2 & 57,5 & 37,5 & 50,7 & 55,3 & 37,5 \\
\hline Linoléico & 0,2 & 0,9 & 26,1 & 0,5 & 0,6 & 24,0 \\
\hline Linolênico & - & 0,3 & 0,5 & - & 0,3 & 0,4 \\
\hline Aracdônico & 0,2 & 0,9 & 0,2 & 0,3 & 0,8 & 0,1 \\
\hline
\end{tabular}

Fonte: LEAT et al. (1964) 
branosos") e 59,7mg/100g para pernil ("semitendinosas"). Os mesmos autores descreveram que a inclusão de 10 ou $20 \%$ de óleo de canola na dieta de suínos não teve efeito significativo no conteúdo de colesterol no músculo. Porém, a gordura corporal apresentou-se com maior concentração de ácidos graxos poliinsaturados.

Há variação no teor de colesterol de acordo com o tipo de corte da carne, quando esta não se encontra crua. Este fato foi justificado por BRAGAGNOLO (1992), que não observou diferença significativa nos teores de colesterol encontrados entre os quatro cortes de carnes cruas, nos quais os valores variaram de $49-54 \mathrm{mg} / 100 \mathrm{~g}$, em base úmida. Na Figura 2, estão representados os níveis de colesterol em diferentes cortes de carne suína crua, em base úmida e em base seca, além do efeito do cozimento. Quando analisados os dados em base seca, o toucinho apresentou teores de colesterol em média de $61 \pm 6 \mathrm{mg} / 100 \mathrm{~g}$, diferindo dos demais cortes. Quando estes cortes foram cozidos, foi encontrada diferença significativa na concentração de colesterol em base úmida, tendo maior concentração de colesterol a bisteca, seguido pelo pernil, lombinho e toucinho. Este último foi o mais baixo devido à perda de gordura após o cozimento. A autora concluiu que com o efeito do cozimento nos diferentes cortes, não houve perdas significativas na concentração de colesterol na bisteca e toucinho, no entanto, ocorreu redução de 12 e $19 \%$ no pernil e lombinho, respectivamente.

\section{CONCLUSÕES}

O colesterol é um nutriente essencial à vida, porém é um fator de risco para aproximadamente $25 \%$ das pessoas acima de 30 anos de idade, as quais apresentam problemas genéticos de regulação do metabolismo deste composto. Indivíduos,

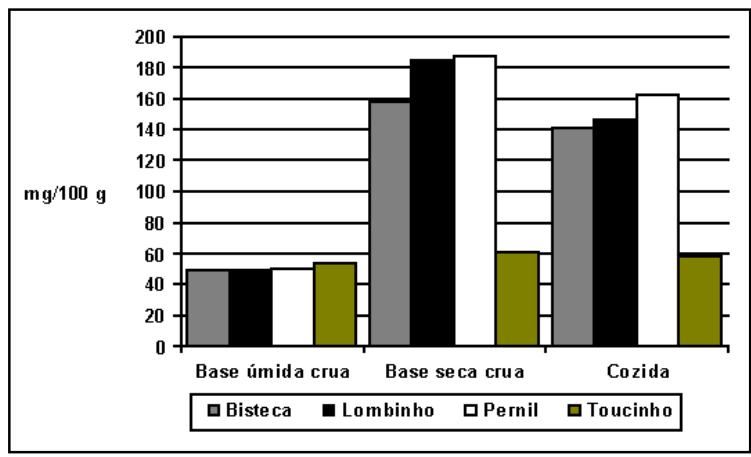

Figura 2 - Concentração de colesterol na carne suína crua em base úmida e em base seca e na carne suína cozida. Fonte: BRAGAGNOLO (1992) com nível de colesterol plasmático acima de $200 \mathrm{mg} / \mathrm{dl}$, mesmo sem doença cardíaca coronariana definida, devem se preocupar com a dieta alimentar contendo principalmente ácidos graxos saturados, como ácido láurico, mirístico e palmítico. No entanto, os ácidos graxos poliinsaturados têm efeito hipocolesterolêmico, tanto para ser humano como para suínos e aves, por aumentar a secreção de ácidos biliares e colesterol na bile pela elevação do número de receptores de LDL na célula, ou por ser convertido, na sua maior parte, em corpos cetônicos. Com isso, é viável suplementar dieta para suínos com ácidos graxos poliinsaturados (gorduras vegetais), porém, em níveis não superiores a $4 \%$ para não obter carcaças com dificuldades de conservação e, por outro lado, ser benéfica para a saúde do consumidor.

\section{REFERÊNCIAS BIBLIOGRÁFICAS}

ANDERSON, J.T., GRANDE, F. KEYS, A Cholesterollowering diets. Journal of the American Dietetic Association, v. 62, p. 133-137, 1973.

ASGHAR, A., LIN, C.F., GRAY, J.I., BUCKLEY, D.J., et al. Effect of dietary oils and $\alpha$-tocopherol supplementation on membranal lipid oxidation in broiler meat. Journal Food Science, v. 55, p. 46-50, 1990 .

BEYNEN, A.C., KATAN, M.B. Why do polyunsaturated fatty acids lower serum cholesterol? American Clinical Nutrition, v. 42 , p. $560-563,1985$.

BOHAC, C.E., RHEE, K. S. Infuence of animal diet and muscle location on cholesterol content of beef and pork muscles. Meat Science, v. 23, p. 71-79, 1988.

BRAGAGNOLO, N. Determinação dos teores de colesterol em carnes, ovos e massas com ovos. Campinas, 1992. 105 p.

BROOKS, C.C. Fatty acid composition of pork lipids as affected by basal diet, fat source and fat level. Journal of Animal Science, v. 33, p. 1224-1231, 1971.

BUDOWSKI, P. Nutritional effects of omega-3 polyunsaturated fatty acids. Journal Medici Science, v. 17, p. 223-231, 1981

CHAMPE, P.C., HARVEY, R.A. Lippincott's illustrated reviews: biochemistry, 2. ed. Philadelphia: Lippincott, 1994 $443 \mathrm{p}$.

COBOS, A., CAMBERO, M.I., ORDÓÑEZ, J.A. Revisión: Influence de la dieta animal en los ácidos grasos de los lipídos de la carne. Revista Española de Ciência y Tecnologia de Alimentos, v. 34, n. 1, p. 35-51, 1994.

CONIGLIO, J.G. How does fish oil lower plasma triglycerides? Nutrition Reviews, v. 50, n. 7, p. 195-206, 1992.

GRUNDY, S.M., VEGA, G.L. Plasma cholesterol responsiveness to saturated fatty acids. American Journal Clinical Nutrition, v. 47, p. $822-824,1988$.

HARPER, A.E. Dietary guidelines challenge animal product consumption. Feedstuffs, August, 2, p. 13-17, 1993.

HOPKINS, P.N. Effects of dietary cholesterol on serum cholesterol : a meta-analysis and review. American Journal Clinical Nutrition, v. 55, p. 1060-1070, 1992. 
IBRAHIM, J.B.T., McNAMARA, D.J. Cholesterol homeostasis in guinea pigs fed saturated and polyunsaturated fat diets. Biochimica et Biophysica Acta, v. 963, p. 109-118, 1988

IRIE, M., SAKIMOTO, M. Fat characteristics of pigs fed fish oil containing eicosapentaenoic and docosahexaenoic acids. Journal of Animal Science, v. 70, p. 470-477, 1992.

KEYS, A. Coronary heart disease in seven countries. Circulation, v. 41, n. 4 (supplement I), 1970.

KINSELLA, J.E., LOKESH, B., STONE, R.A. Dietary n-3 polyunsaturated fatty acids and amdioration of cardiovascular disease: Possible mechanisms. American Journal Clinical Nutrition, v. 52, p. 1-28, 1990.

KRIS-ETHERTON, P.M., KRUMMEL, D., RUSSELL, M.E., $\boldsymbol{e}$ al. The effect of diet on plasma lipids, lipoproteins and coronary heart disease. Journal of the American Dietetic Association, v. 88, p. $1373,1988$.

LEAT, W.M.F., CUTHBERTSON, A., HOWARD, A. N., et al Studies on pigs reared on semi-synthetic diets containing no fat, beef tallow and maize oil: composition of carcass and fatty acid composition of various depot fats. Journal Agriculture Science, v. 63, p. 311-317, 1964.

MILES, R.D. Eggs important in diet, unfairly criticized as heart disease risk. Feedstuffs, september, p. 26-51, 1989.

MILLER, M.F., SHACKELFORD, S.D., HAYDEN, K.D., et al. Determination of the alteration in fatty acid profiles, sensory characteristics and carcass traits of swine fed elevated levels of monounsaturated fats in the diet. Journal of Animal Science, v. 68, p. 1624-1631, 1990.

MORGAN, C.A., NOBLE, R.C., COCCHI, M., et al. Manipulaion of the fatty acid composition of pig meat lipids by dietary means. Journal Science Food Agriculture, v. 58, p. $357-368,1992$

MYER, R.O., LAMKEY, J.W., KNAUFT, D.A., $\boldsymbol{e}$ t $\boldsymbol{a}$. Performance and carcass characteristics of swine when fed diets contaning canola oil and added copper to alter the unsaturated:saturated ratio of pork fat. Journal of Animal Science, v. 70, p. 1417-1423, 1992.

NABER, E.C. The cholesterol problem, the egg and lipid metabolism in the laying hen. Poultry Science, v. 55, p. 1430,1976

NATIONAL CHOLESTEROL EDUCATION PROGRAM Report of the national cholesterol education program expert panel on detection, evaluation, and treatment of high blood cholesterol in adults. Archives of Internal Medicine, v. 148, p. 36,1988

NORUM, K.R. Dietary fat and blood lipids. Nutrition Reviews, v. 50, n. 4 , p. 30-37, 1992.
PARK, J., RHEE, K.S., KEETON, J.T., et al. Properties of lowfat frankfurters containing monounsaturated and omega-3 polyunsaturated oils. Journal Food Science, v. 54, p. 500$504,1989$.

REISER, R. O colesterol dos ovos e a saúde humana. Avicultura e Suinocultura Industrial, v. 79, n. 952, p. 57-61, 1989.

REITMEIER, C.A., PRUSA, K.J. Cholesterol content and sensory analysis of ground pork as influenced by fat level and heating. Journal of Food Science, v. 52, n. 4, p. 916, 1987.

RHEE, K.S., ZIPRIN, Y.A., ORDONEZ, G., et $\boldsymbol{a l}$. Fatty acid profiles and lipids oxidation in pork muscles as affected by canola oil in the animal diet and muscle location. Meat Science, v. 23, p. 201-210, 1988.

RHEE, K.S., ZIPRIN, Y.A., DAVIDSON, T.L. Characteristics of pork products from swine fed a high monounsaturated fat diet : part 2 - uncured processed products. Meat Science, v. 27, p. 343-357, 1990.

SHERF, H., BIEBER-WLASCHNY, M. How nutrition influences fat quality. Pig International, v. 20 , n. 2, p. 14 -18, 1990.

SILVA, G.J.C. Colesterol : O mito do colesterol e doenças coronarianas em poedeiras e no homem. Avicultura Ciência \& Tecnologia, ano 3, n. 15, p. 2-4, 1995

SKELLEY, G.C., BORGMAN, R.F., HANDLIN, D.L., $\boldsymbol{e}$ t al. Influence of diet on quality, fatty acids and acceptability of pork. Journal of Animal Science, v. 41, p. 1298-1304, 1975.

SMITH, R.L., PINCKNEY, E.R. The cholesterol conspiracy, Missouri, Wanen H. Green, Inc., 1991, 388 p.

STEHBENS, W.E. Lecture presented at the cardiology department, Cedars Sinai Hospital, March 28, 1989.

STURDEVANT, R.A.L., PEARCE, M.L., DAYTON, S. Increased prevalence of choletithiasis in men ingesting a serum cholesterol-lowering diet. N. Engl. Journal Medicine, v. 288 , p. $24-27,1973$

VILLEGAS, F.J., HEDRICK, H.B., VEUM, T.L., et al. Effect of diet and fatty acid composition of porcine adipose tissue. Journal of Animal Science, v. 36, p. 663-668, 1973

WEINTRAUB, M.S., ZECHNER, R., BROWN, A., et al. Dietary polyunsaturated fats of the $\mathrm{W}$ - and W-3 series reduce postprandial lipoprotein levels. Chronic and acute effects of fat saturation on postprandial lipoprotein metabolism. Journal Clinical Invest, v. 82, p. 1884-1893, 1988.

ZIPRIN, Y.A., RHEE, K.S., DAVIDSON, T.L. Characteristics of pork products from swine fed a high monunsaturated fat diet : part 3 - a high-fat cured product. Meat Science, v. 28, p. 171$180,1990.127$

Ciência Rural, v. 29, n. 1, 1999. 